\title{
ENHANCEMENT OF ORGAN OF INTEREST VIA BACKGROUND SUBTRACTION IN CONE BEAM ROTATIONAL ANGIOCARDIOGRAM
}

\author{
Mingqing Chen ${ }^{1}$, Yefeng Zheng ${ }^{1}$, Kerstin Mueller ${ }^{2,3}$, Christopher Rohkoht ${ }^{2}$, \\ Guenter Lauritsch ${ }^{2}$, Jan Boese ${ }^{2}$, Dorin Comaniciu ${ }^{1}$ \\ ${ }^{1}$ Image Analytics and Informatics, Siemens Corporate Research, USA \\ ${ }^{2}$ Healthcare Sector, Siemens AG, Forchheim, Germany 91301 \\ ${ }^{3}$ Pattern Recognition Lab, University Erlangen-Nuremberg, Germany \\ yefeng.zheng@siemens.com
}

\begin{abstract}
The real time X-ray angiography based on $\mathrm{C}$-arm cone beam system is the workhorse imaging modality for interventional cardiac procedures. These images are two-dimensional (2D) projections of three dimensional (3D) objects along the X-ray direction. The organ of interest (OOI), such as left ventricle (LV) endocardium, in the projection image is superimposed with other anatomical structures and often has low contrast. In this study, a novel approach is proposed to isolate the OOI in projection images by subtracting with a background image, which is generated by numerical projection of 3D tomographic image with OOI masked out. Study based on one patient and one pig image is taken. About two to three-fold increase in the contrast-to-noise ratio (CNR) is achieved for LV endocardium, compared to an unprocessed image.
\end{abstract}

Index Terms - Subtraction, CBCT, SART, Reprojection, Ventricle

\section{INTRODUCTION}

Currently, the interventional cardiac procedures are typically performed using C-Arm based X-ray angiography systems. The systems provide high temporal resolution of twodimensional (2D) projection images, which are used for visual guidance during the procedures. Those $2 \mathrm{D}$ projection images could also be used off-line to analyze the cardiac motion. It would be desirable if one can derive some clinically significant parameters from the $2 \mathrm{D}$ projection images, such as the volume of left ventricle (LV) as a function of time, the ejection fraction rate and the regional wall motion. However, the $2 \mathrm{D}$ fluoroscopic images contain no depth information as the nature of $3 \mathrm{D}$ to $2 \mathrm{D}$ perspective projection. The organ of interest $(\mathrm{OOI})$, such as the $\mathrm{LV}$, in $2 \mathrm{D}$ projection images is often superimposed with other anatomic structures, making it less distinguishable. This feature of 2D projection images limits their use for tissue localization and boundary delin- eation. Though recent development of ECG-gated 4D cardiac C-Arm CT has combined the advantages of both spatial and temporal resolution [1], which makes it feasible for tissue segmentation under different cardiac phases, it requires excessive scanning time and imaging dose as a result of using multiple sweeps of C-arm.

Background subtraction has been very popular in video motion enhancement. It was also experimented in X-ray fluoroscopic images for scenarios with fixed X-ray source and imaging detector, such as subtracting the average of neighboring frames for motion enhancement in tumor detection [2]. For rotational fluoroscopic systems, digital subtraction angiography (DSA) is produced by acquiring images before and after the administration of contrast agents and subtracting the results. However, two times of sweeps are needed and the Carm motion and the cardiac motion need to be synchronized to minimize the mis-alignment of the pre-contrast image and contrasted image.

In this work, we propose a novel technique called OOI based tomographic imaging via background subtraction (OOI-TIBS) to enhance the LV area in 2D fluoroscopic images. OOI-TIBS aims to remove the background structuresi.e. not belonging to the OOI (LV endocardium in this study)along the path of X-ray projection. Firstly, a 3D volume is reconstructed from $2 \mathrm{D}$ cone beam projections. Gated reconstruction can be applied in this step, where only a subset of projections that belong to a particular cardiac phase is used. Then the OOI region of the 3D volume is masked with zero intensity. The masked image is numerically projected onto each 2D projection, which is called "background image". It is used to subtract the original $2 \mathrm{D}$ projection to produce a subtracted image, which is anticipated to have enhanced OOI region. Our approach is significantly different from TIBS proposed by Zhang et al. [3], where the original 3D volumetric data and the re-projected 2D images are based on the same cone beam $\mathrm{C}$-arm system. And we aim to enhance a particular organ (LV) in the projection image, not one single 
slice of volumetric data.

\section{METHODS}

\subsection{General Workflow of OOI-TIBS Technique}

Consider a flat panel detector receiving attenuated X-ray from the source. Under the ideal situation where no scattering of the photons occurs, the detected intensity of projection images at pixel location $(u, v)$ is given by

$$
I(u, v)=I_{0} e^{-P(u, v)},
$$

where $I_{0}$ is the incident intensity in the absence of scan object and $P$ is the primary beam attenuation, which is equal to the line integral of the linear attenuation coefficient

$$
P(u, v)=\int_{\vec{r} \in L} \mu(\vec{r}) d \vec{r} .
$$

The line integral along $L$ of 3D tomographic image can be divided into OOI region $L_{o}$ and background (non-OOI) region $L_{b}$,

$$
P(u, v)=\int_{\vec{r} \in L_{o}} \mu(\vec{r}) d \vec{r}+\int_{\vec{r} \in L_{b}} \mu(\vec{r}) d \vec{r} .
$$

In transmission imaging, the $\mathrm{X}$-rays reaching the detector consist of unscattered (primary) and scattered (secondary) components. Numerical projection differs from real projection that scattered component is not considered. For a typical $\mathrm{kV}$ CBCT image on human anatomy, the scatter-to-primary ratio is greater than $100 \%$, leading to CT number inaccuracies on the order of $40 \%$ [4]. Besides, the tissue outside the field of view (FOV) of volumetric image is not accounted during the projection. Theoretically, one can also directly perform numerical projection of the OOI region $\int_{\vec{r} \in L_{o}} \mu(\vec{r}) d \vec{r}$. The major problem of applying this method is the cardiac motion. Motion compensated reconstruction technique is needed in order to derive a $3 \mathrm{D}$ volume with reduced motion artifact. However, since residual motion could still exist in the reconstructed volume, direct projection may result in deviation from the original $2 \mathrm{D}$ OOI region, such as the position, the shape and the size.

Fig. 1 shows the general workflow of the OOI-TIBS approach. Fig. 1a is the original 2D projection images displayed in projected intensity $P$. The projection images are sorted into four different cardiac phases according to ECG signal. ECG-gated volume reconstruction is performed to generate $3 \mathrm{D}$ volumetric images under four different phases based on the SART algorithm. The epicardium and endocardium of the LV is automatically localized and segmented the marginal space learning using method [5]. Fig. 1b shows the 3D visualization of the reconstructed volume overlaid with the mesh of segmented LV endocardium. Since the LV dilates and contracts constantly over the course of image acquisition, the OOI region should be large enough to encompass LV endocardium in all the phases. We use the LV epicardium as the

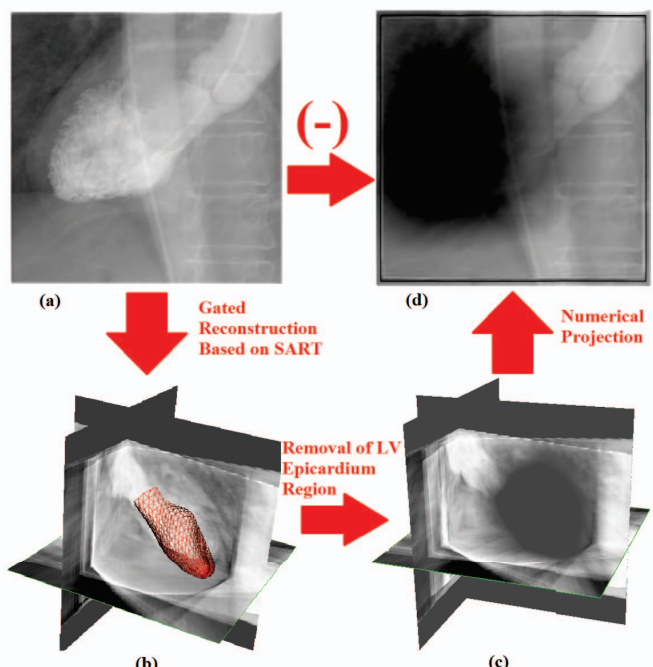

(b)

(c)

Fig. 1. The workflow of OOI-TIBS approach

OOI region. The linear attenuation coefficient of all the voxels inside this region is set zero, thus leaving only background structures. To make the transition to zero smooth, the voxels close to the LV boundary is also reduced by a certain amount, which is in inverse relationship to the distance to the boundary. The remained tomographic image after OOI removal is used to generate numerical projection in corresponding orientation angle. Fig. 1c shows the 3D background volume with removed OOI region. Fig. 1d shows the numerical projection of the background image. Fig. 2a shows the background subtraction result of Fig. 1a and Fig. 1d. All the three images are displayed using the same intensity window setting. We can observe that the tubular structures (the ascending and descending aortas) that originally exist in the unprocessed image disappear now.

\subsection{Data}

OOI-TIBS was tested on two real sets of projection images, including a patient and a pig. For the patient dataset, about five cardiac cycles were captured in the image sequence with 133 projection images. The frame rate of the pig dataset was set much higher, resulting in 395 projection images, spanning 13 cardiac cycles. Both of the patient and pig was injected with contrast agents in the LV prior to the image acquisition. The ECG signal is recorded to assign a cardiac phase to each $2 \mathrm{D}$ projection. The gated reconstruction was applied for both patient and pig data. Four phases were applied, resulting in about 30 and 100 projection images used for each phase for the patient and pig, respectively. The dimension of the 3D volume was set at $256 \times 256 \times 256$. The diameter of FOV cylinder for patient and pig is about $100 \mathrm{~mm}$ and $200 \mathrm{~mm}$ respectively. So more truncation occurs for patient data. 


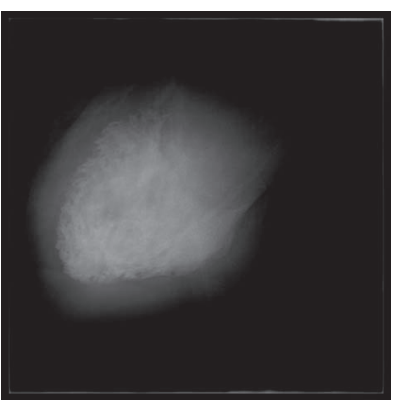

(a)

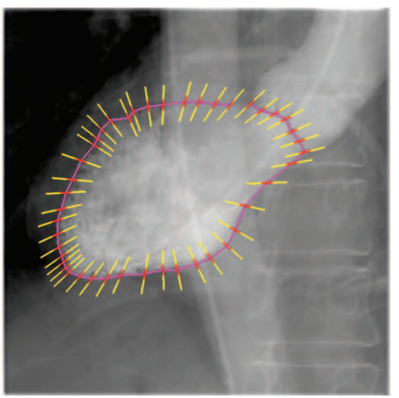

(b)

Fig. 2. (a) 2D projection image after background subtraction; (b) An illustration of using profile along LV boundary to calculate the contrast-to-noise ratio (CNR).

\subsection{Numerical Projection Based on SART}

The image reconstruction of OOI-TIBS is based on simultaneous algebraic reconstruction technique (SART) [6]. The computation of ray integral of attenuation coefficients based on image reconstructed by SART is inherently more authentic than the traditional FDK algorithm, since it converges to the 2D projection data during the iterations (4). Compared to ART, SART has no stripping nor aliasing artifact [7]), which is more suitable for cone beam reconstruction.

SART updates the linear attenuation coefficient of each voxel by the following equation,

$$
v_{j}^{k}=v_{j}^{(k-1)}+\lambda \frac{\sum_{\rho_{i} \in P_{\varphi}} \frac{\left(p_{i}-\sum_{n=1}^{N} w_{i n} v_{n}^{(k-1)}\right)}{\sum_{n=1}^{N} w_{i n}} w_{i j}}{\sum_{\rho_{i} \in P_{\varphi}} w_{i j}} .
$$

The equation shows how to update the $j^{t h}$ voxel from $(k-$ $1)^{t h}$ iteration to $k^{t h}$ iteration. The numerator of the correction term on the right is the difference between original pixel value $p_{i}$ and the numerically projected value $\sum_{n=1}^{N} w_{i n} v_{n}^{(k-1)}$, which is the line integral along the path of the X-ray. The correction term depends on a weighted average of all rays of projection $P_{\varphi}$ that traverse the voxel $j$, where $\varphi$ denotes the orientation angle at which the projection is taken.

\subsection{Computation of Line Integral under Cone Beam Ge- ometry}

To compute the voxel's contribution $w_{i j} v_{j}$ to $P_{i}$ in Equation (4), an interpolation kernel $h$ is needed for the continuous volume representation

$f(x, y, z)=\sum_{n_{x}} \sum_{n_{y}} \sum_{n_{z}} f\left(n_{x}, n_{y}, n_{z}\right) h\left(x-n_{x}, y-n_{y}, z-n_{z}\right)$.

Here $x, y, z$ and $n_{x}, n_{y}, n_{z}$ is the continuous and discrete Cartesian coordinate representation of the volumetric image respectively. Among various choices of $h$, we use a kernel based on the Kaiser-Bessel window, which has many good features such as fast decay for frequencies past the Nyquist rate and radial symmetry. The line integral as a function of distance to voxel center is pre-computed analytically into a kernel footprint. During the re-projection and back-projection process, we only need to compute the distance between the voxel and X-ray. Generally an X-ray passing through the volume can be represented as following

$$
\vec{x}(t)=\vec{s}+\overrightarrow{d t}
$$

where $\vec{s}$ is the position of the X-ray source in room coordinate at one orientation angle; $\vec{d}$ is an $R^{3}$ direction vector of the ray, which is usually normalized; $t$ is a parameter indicating the distance to the source. The source position can be determined from the $3 \times 4$ projection matrix $\mathbf{P}$ used in $\mathbf{C}$-Arm system,

$$
\vec{s}=-\mathbf{P}_{3}^{-1} \mathbf{p}_{4}
$$

where $\mathbf{P}_{\mathbf{3}}$ is the matrix containing the first three columns of $\mathbf{P}$ and $\mathbf{p}_{\mathbf{4}}$ is the fourth column. The direction vector can be computed from $2 \mathrm{D}$ projected position $[u, v]$,

$$
\vec{d}=-\mathbf{P}_{3}^{-1} \mathbf{p}^{+}
$$

where $\mathbf{p}^{+}=[u, v, 1]^{T}$. The distance between one voxel center and an X-ray can be computed as

$$
\|r\|=\left\|\vec{d} \times\left(\vec{s}-\overrightarrow{v_{j}}\right)\right\|
$$

Here $\overrightarrow{v_{j}}$ is the center position of voxel $v_{j}$.

\section{EXPERIMENTS}

To evaluate the performance of OOI-TIBS, we explore the intensity change along the boundary of LV endocardium in both unprocessed images and images after background subtraction. The boundary is manually contoured by a clinician. Relative contrast-to-noise ratio (CNR) is a simple and objective measure of the detectability of certain structures with uniform intensity. Here CNR is used to measure the intensity profiles inside and outside the region of the LV endocardium, which is defined as $C N R=\left(I_{\text {in }}-I_{\text {out }}\right) / \sqrt{\sigma_{\text {in }}+\sigma_{\text {out }}}$, where $I$ and $\sigma$ represents the average and standard deviation of profile intensities and the subscript in and out represents inside or outside of the LV endocardium region. An example of the intensity profile is shown in Fig. 2b. For each contour point, we define the profile (yellow) as a ling segment along the radial direction of the contour shape (purple). A margin (red) is set to allow some error of manual annotation. It should be noted that for visualization purpose, the length of the profile shown in the picture is longer than the actual length used in validation.

Fig. 3 shows the improvement of CNR in both patient and pig images in all the projection images. For the patient images, the averaged CNR in all unprocessed images is 0.142 , 
with a standard deviation 0.144. After the background subtraction, the CNR increases to 0.274 , with a standard deviation 0.198. For the pig images, the averaged CNR increases from $0.287 \pm 0.290$ to $0.694 \pm 0.571$. For comparison, the OOI region size is also plotted. It can be observed that CNR improvement is larger when the OOI region size is large, which represents the cardiac phase near end-diastolic. During this phase more contrast agents are filled into left ventricle, generating better contrast for both unprocessed and backgroundsubtracted images.

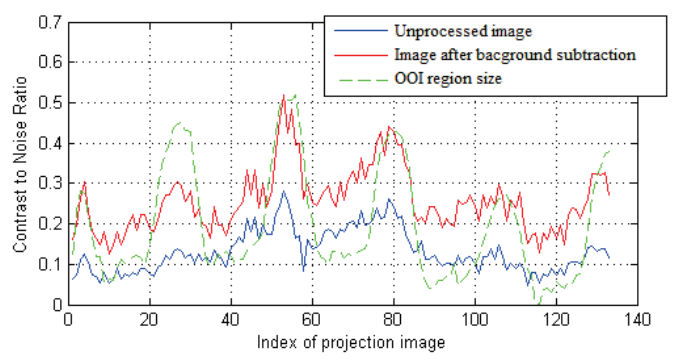

(a)

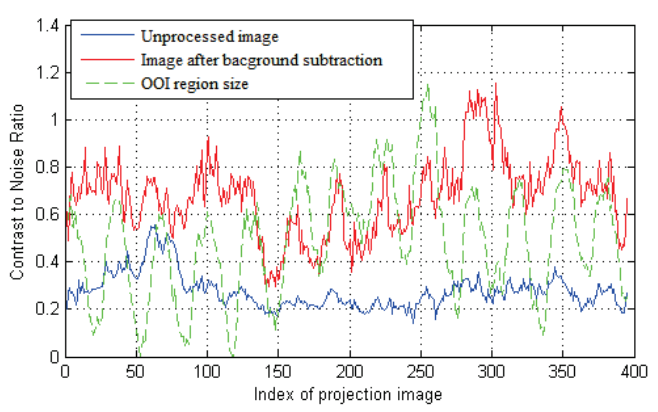

(b)

Fig. 3. Contrast-to-noise ratio (CNR) against the index of projection images (a) Patient image sequence; (b) Pig image sequence.

Fig. 4 compares one pig image after background subtraction with unprocessed image. CNR increases from 0.201 to 0.614 . It can be observed that many superimposed structures are removed in the result images.

\section{CONCLUSION}

In this paper, we propose a novel technique (OOI-TIBS) to enhance the contrast of the left ventricle endocardium in 2D projection images of $\mathrm{C}$-arm rotational angiography by subtracting the background information. No extra scanning or modification to the image acquisition operation is required. Results based on one patient and one pig image shows significant improvement on CNR and effective reduction of superimposed anatomical structures. This technique would be greatly helpful to quantitative evaluation of cardiac functions

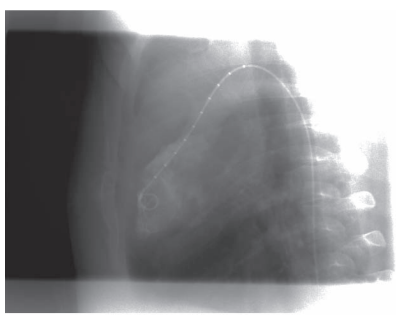

(a)

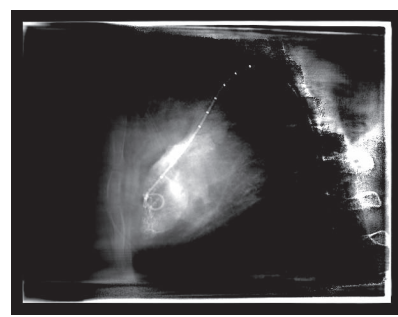

(b)
Fig. 4. Background subtraction results. (a) Unprocessed pig image of frame 1; (b) image after background subtraction

based on 2D projection images.

\section{Acknowledgments:}

We would like to thank to Drs. Patrick W. Serruys, Carl Schultz, Peter de Jaegere and Robert van Geuns, Thorax Center, Erasmus MC, Rotterdam, The Netherlands, and Drs. Rebecca Fahrig and Erin Girard, Department of Radiology, Stanford University, USA, for acquiring patient and animal data, respectively.

\section{REFERENCES}

[1] G. Lauritsch, J. Boese, L. Wigstrom, H. Kemeth, and R. Fahrig, "Towards cardiac C-arm computed tomography," IEEE Trans. Medical Imaging, vol. 25, no. 7, pp. 922 -934, July 2006.

[2] R.I. Berbeco, H. Mostafavi, G.C. Sharp, and S.B. Jiang, "Towards fluoroscopic respiratory gating for lung tumours without radiopaque markers," physics in Medicine and Biology, vol. 50, pp. 4481, 2005.

[3] J. Zhang, B. Yi, G. Lasio, M. Suntharalingam, and C. Yu, "Tomographic image via background subtraction using an $\mathrm{x}$-ray projection image and a priori computed tomography," Medical physics, vol. 36, pp. 4433, 2009.

[4] J.H. Siewerdsen and D.A. Jaffray, "Cone-beam computed tomography with a flat-panel imager: magnitude and effects of X-ray scatter," Medical physics, vol. 28, pp. 220, 2001.

[5] Y. Zheng, A. Barbu, B. Georgescu, M. Scheuering, and D. Comaniciu, "Four-chamber heart modeling and automatic segmentation for 3D cardiac CT volumes using marginal space learning and steerable features," IEEE Trans. Medical Imaging, vol. 27, no. 11, pp. 1668-1681, 2008.

[6] A. H. Andersen and A. C. Kak, "Simultaneous algebraic reconstruction technique (SART): A superior implementation of the ART algorithm," Ultrasonic Imaging, vol. 6, no. 1, pp. 81-94, 1984.

[7] Klaus Mueller, Roni Yagel, and John J. Wheller, "Anti-aliased three-dimensional cone-beam reconstruction of low-contrast objects with algebraic methods," IEEE Trans. Med. Imag, vol. 18, pp. 519-537, 1999. 